\title{
Seroprevalence of Crimean Congo Hemorrhagic Fever Virus in ljara District, Kenya
}

\author{
Olivia Wesula Lwande,,4 Zephania Irura, ${ }^{2}$ Caroline Tigoi,, Edith Chepkorir, Benedict Orindi, \\ Lillian Musila, ${ }^{3}$ Marietjie Venter, ${ }^{4,5}$ Anne Fischer, and Rosemary Sang ${ }^{1,3}$
}

\begin{abstract}
Crimean-Congo hemorrhagic fever (CCHF) is a tick-borne viral disease mainly affecting pastoralists who come in contact with animals infested with Hyalomma ticks, which are the key vectors of CCHF virus (CCHFV). CCHFV has been detected among these ticks in parts of North Eastern Kenya. This study aimed to identify acute cases of $\mathrm{CCHF}$, and to determine the extent of previous exposure to CCHFV in an outpatient population attending Sangailu and Ijara health centers, Ijara District, North Eastern Kenya, presenting with acute febrile illnesses. A total of 517 human serum samples were collected from these patients. The samples were screened for the presence of IgM and IgG antibodies to CCHF using CCCHF-IgG and IgM ELISA test kits. A multivariable logistic regression model was used to investigate the risk factors associated with evidence of exposure to CCHFV. A single patient tested positive for anti-CCHF IgM, while 96 were positive for anti-CCHF IgG. The seroprevalence of CCHFV was 23\% in Sangailu and 14\% in Ijara. Most exposed persons were aged 40-49 years. The likelihood of exposure was highest among farmers (29\%). Age, location, and contact with donkeys were significantly associated with exposure to CCHFV. Acute CCHFV infections could be occurring without being detected in this population. This study confirms human exposure to CCHF virus in Ijara District, Kenya, and identifies several significant risk factors associated with exposure to CCHFV.
\end{abstract}

Key Words: Crimean-Congo hemorrhagic fever-Hyalomma—Ijara District, Kenya-Ticks.

\section{Introduction}

C Rimean-Congo hemorrhagic Fever (CCHF) is a disease caused by a virus of the Nairovirus genus, family Bunyaviridae. The disease is endemic in many countries in Europe, Asia, and Africa (Swanepoel, 2005). It was first reported in the Crimean peninsula in the mid-1940s, when a large outbreak of severe hemorrhagic fever occurred with a case fatality rate of $>30 \%$ (Hoogstraal 1979; Nichol et al., 2001), and was designated as Crimean hemorrhagic fever. The virus was later shown to be antigenically identical to Congo virus isolated from a febrile patient in the Democratic Republic of Congo in 1956 (Hoogstraal 1979), and was subsequently renamed Crimean-Congo hemorrhagic fever virus (CCHFV; Casals 1969).

Members of the tick genus Hyalomma are the principal vectors of CCHFV. There is evidence that the virus causes infection in birds, such as ostriches, guinea fowls, and hornbills (Hoogstral 1979), and that antibodies against CCHFV have been detected in the sera of horses, donkeys, goats, cattle, sheep, and pigs, in various regions of Europe, Asia, and Africa (Shimshony 2010). Birds and domesticated ruminants including cattle, sheep, and goats, become viremic for 1 week after experimental infection (Shimshony, 2010).

Humans become infected through a bite of or crushing an infected tick with bare skin with an open wound, by contact with animal blood or tissues, and by drinking unpasteurized milk from infected animals; aerosol transmission has also been documented in Russia (Shimshony 2010). Possible horizontal transmission has also been reported from a mother to her child in Russia (Shimshony 2010).

The signs and symptoms of CCHF disease/infection include hemorrhagic manifestations such as ecchymosis, petechia, epistaxis, hematemesis, and hemorrhagic enteritis, as

\footnotetext{
${ }^{1}$ Department of Human Health, International Centre for Insect Physiology and Ecology, Nairobi, Kenya.

${ }^{2}$ Ministry of Public Health and Sanitation, National Laboratory Surveillance and Response, Nairobi, Kenya.

${ }^{3}$ U.S. Army Medical Research Unit, Nairobi, Kenya.

${ }^{4}$ Department of Medical Virology, University of Pretoria, Pretoria, Sandringham, South Africa.

${ }^{5}$ National Institute for Communicable Diseases, Pretoria, Sandringham, South Africa.
} 
reflected by bloody diarrhea. This is accompanied by one or more of the following symptoms: fever, severe headache, joint pain, chills, and nausea (Elata et al., 2011).

Epidemiologically, CCHF cases are distributed mainly among rural actively-working persons exposed to tick bites (Gao 2010), with the highest-risk group being livestock farmers living in endemic areas. Almost $90 \%$ of the cases in a recent outbreak in Turkey were farmers (Shimshony 2010). Abattoir workers who work with large domestic animals are also at risk, through either slaughtering viremic animals, or exposure to ticks that fall from the animal hides. Ingestion of meat itself is not a risk because the virus is inactivated by postslaughter acidification of the tissues. Gender distribution varies between countries, depending on the relative participation of men and women in agricultural work (Shimshony 2010).

The geographic range of CCHFV is the most extensive of the tick-borne viruses affecting humans (Ergonul 2006). In Kenya, CCHFV has been detected in ticks on only two occasions: in a pool of Rhipicephallus pulchellus ticks collected in the 1970s from a dying sheep in Kabete veterinary laboratory (Hoogstraal 1979), and most recently in multiple pools of Hyalomma ticks collected in North Eastern Kenya (Sang et al. 2011). However, only one human case of CCHFV infection has been documented in western Kenya in October 2000, which represents the first and only documented case of acute human CCHFV infection in Kenya (Dunster 2002).
Although evidence of circulation of CCHFV among ticks suggests that humans may be exposed in parts of Kenya, CCHFV transmission among the human population has not been well characterized. In this study, serum samples collected from acute febrile illness surveillance patients in Ijara District, a pastoralist community in rural northern Kenya, were screened for the presence of both anti-CCHF IgM and IgG antibodies to determine the extent of CCHFV circulation among the human population. IgM antibodies are a hallmark of acute infection, whereas IgG antibodies are present in convalescent phase or indicate previous exposure to the infection.

This study aimed to identify acute cases of CCHF disease and to determine the extent of previous exposure to CCHFV in an outpatient population attending Sangailu and Ijara health centers, Ijara District, North Eastern Kenya, with complaints of acute febrile illnesses.

\section{Materials and Methods}

\section{Study area}

The study was conducted in Ijara District of the North Eastern province of Kenya (Fig. 1). This is an arid and semiarid region where $90 \%$ of the people practice nomadic pastoralism, keeping indigenous cattle, goats, sheep, donkeys, and camels. Prolonged dry seasons trigger the movement of people and livestock to the Tana River Delta and the Boni

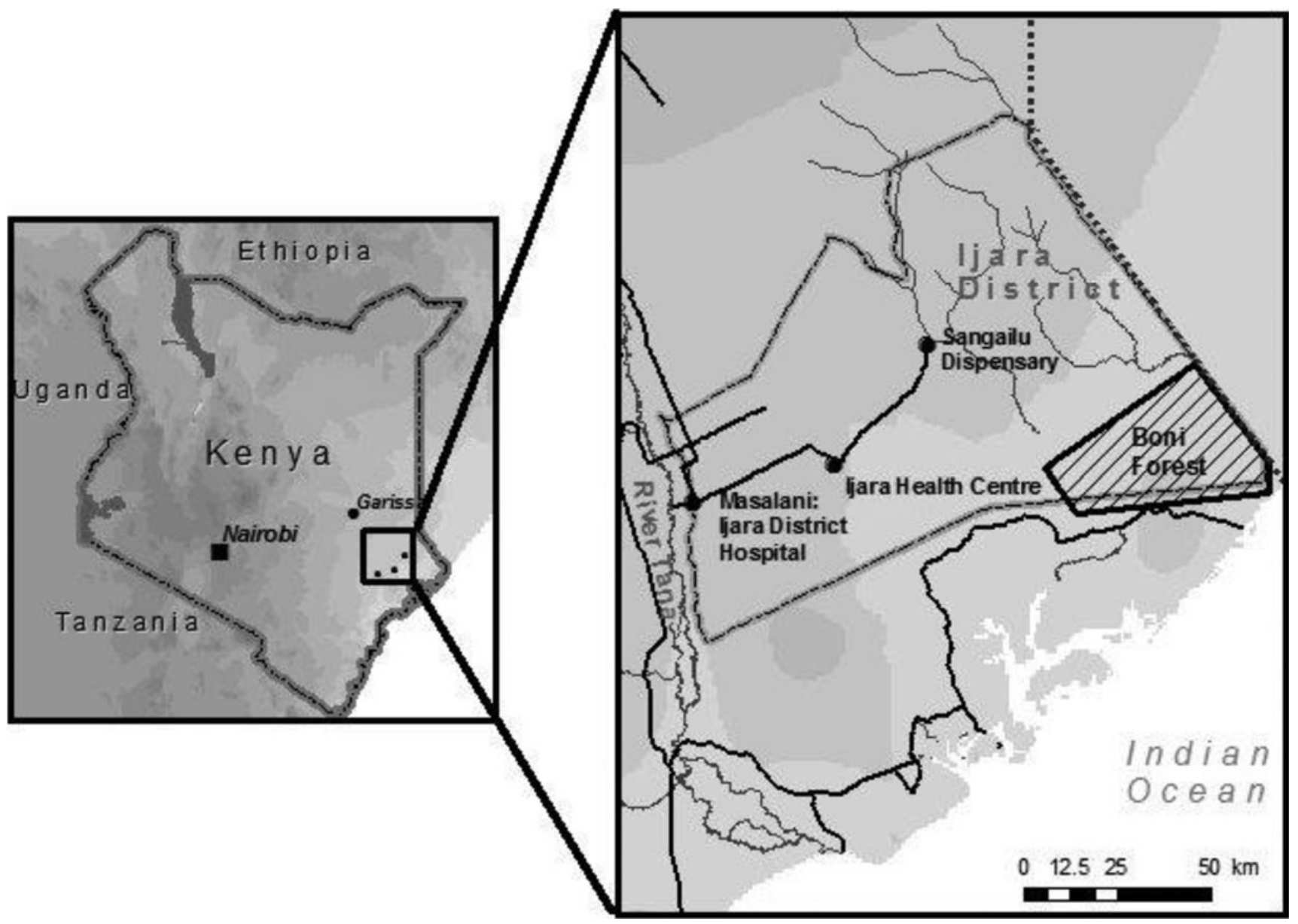

FIG. 1. A map of the study sites in Ijara District in North Eastern Province, Kenya. 
forest area near the Indian Ocean coastline, where water and pasture are abundant long after the rains have gone. This migration pattern facilitates the movement of potentially infected ticks across great distances, presenting an opportunity for the exchange of diverse tick species and populations among huge animal and human populations, and hence the risk of exposure to tick-borne disease.

This was a descriptive, cross-sectional, and laboratorybased study conducted between October 2010 and March 2011. Blood samples were obtained from patients presenting with acute febrile illnesses, with complaints including fever, chills, cough, headache, joint aches, general body weakness, and any hemorrhagic signs.

\section{Ethical considerations}

The samples used in this study were de-identified, and only study codes were used, with no reference to patient names on the sample vials. Data and samples were collected only from consenting patients. Approval to carry out the study was obtained from the Kenya Medical Research Institute (KEMRI) National Ethical Review Committee.

\section{CCHFV antibody testing}

Detection of all CCHF virus-specific IgM and IgG antibodies was performed with the indirect ELISA format previously described by Harun and associates (2010). This was done using a commercial kit that came with its own internal negative and positive controls (VectoCrimean-CHF-IgG and IgM ELISA test kits; Vector-Best, Novosibirsk, Russia). The sensitivity to the standard panel of positive samples is indicated as $100 \%$, and the specificity of the standard panel of negative samples $100 \%$.

\section{IgM capture ELISA}

All samples were heat inactivated at $56^{\circ} \mathrm{C}$ for $30 \mathrm{~min}$. Inactivated lysates of tissue culture cells infected with the CCHF virus, and a similarly diluted mock antigen made from uninfected cells, were used as positive and negative antigens, respectively. A CCHF virus-positive human serum served as positive control serum, and serum negative by ELISA for CCHF virus-specific antibodies served as a negative control. HRPconjugated anti-human Fc-specific IgM diluted in serum dilution buffer was used as a conjugate, and ABTS peroxidase parts A and B combined 1:1 was used as a substrate. Wash buffer was phosphate-buffered saline. One-half of the plate was coated with $100 \mu \mathrm{L}$ per well of positive antigen diluted at 1:1000. The second half of the plate was coated with $100 \mu \mathrm{L}$ per well of mock control antigen diluted 1:1000. The plates were incubated in a moist environment overnight at $2-8^{\circ} \mathrm{C}$, then washed three times with $300 \mu \mathrm{L}$ of wash buffer. Then $100 \mu \mathrm{L}$ of sample serum (as well as positive and negative controls) were added to both positive and negative wells at 1:100 dilution (hence each sample was tested against positive and negative antigens), incubated for $1 \mathrm{~h}$ in moist conditions at $35-37^{\circ} \mathrm{C}$, and then washed three times with $300 \mu \mathrm{L}$ per well of wash buffer. Then $100 \mu \mathrm{L}$ of diluted HRP labeled with anti-human IgM antibody was added, and the plates were incubated for $1 \mathrm{~h}$ at $35-37^{\circ} \mathrm{C}$. Then $100 \mu \mathrm{L}$ of the ABTS peroxidase substrate was added to each well. The plates were incubated for $30 \mathrm{~min}$ at $35-37^{\circ} \mathrm{C}$, and then read spectrophotometrically at $405 \mathrm{~nm}$ on an ELISA reader.

\section{Indirect IgG capture ELISA}

All samples were heat inactivated at $56^{\circ} \mathrm{C}$ for $30 \mathrm{~min}$. Inactivated lysates of tissue culture cells infected with the CCHF virus and a similarly diluted mock antigen made from uninfected cells were used as positive and negative antigens, respectively. A positive anti-CCHF IgG human serum served as positive control serum, and serum negative by ELISA for CCHF IgG-specific antibodies served as a negative control. HRP-conjugated anti-human Fc-specific IgG diluted in serum dilution buffer was used as a conjugate, and ABTS peroxidase parts $\mathrm{A}$ and $\mathrm{B}$ combined 1:1 was used as a substrate. Wash buffer was phosphate-buffered saline. One-half of the plate was coated with $100 \mu \mathrm{L}$ per well of positive antigen diluted at $1: 1000$. The second half of the plate was coated with $100 \mu \mathrm{L}$ per well of mock control antigen diluted at 1:1000. The plates were incubated in a moist environment overnight at $2-8^{\circ} \mathrm{C}$, then washed three times with $300 \mu \mathrm{L}$ of wash buffer. Then $100 \mu \mathrm{L}$ of sample serum (as well as positive and negative controls) were added to both positive and negative wells at 1:100 dilution (hence each sample was tested against both positive and negative antigens), incubated for $1 \mathrm{~h}$ in moist conditions at 35$37^{\circ} \mathrm{C}$, and then washed three times with $300 \mu \mathrm{L}$ per well of wash buffer. Then $100 \mu \mathrm{L}$ of diluted HRP labeled with anti-human IgG antibody was added and the plates were incubated for $1 \mathrm{~h}$ at $35-37^{\circ} \mathrm{C}$. Finally, $100 \mu \mathrm{L}$ of the ABTS peroxidase substrate was added to each well. The plates were incubated for $30 \mathrm{~min}$ at $35-37^{\circ} \mathrm{C}$, and read spectrophotometrically at $405 \mathrm{~nm}$ on the ELISA reader.

\section{Interpretation of the results of both IgM and IgG ELISAs}

The adjusted OD was calculated by subtracting the OD of the negative antigen-coated wells from the positive antigencoated wells. The OD cut-off was calculated as the mean of the adjusted OD of the negative control serum plus three times the standard deviation, generally an OD of $\leq 0.2$ at a 1:100 sample dilution. A serum sample was considered positive if the adjusted OD value was greater than or equal to the assay cut-off or 0.2 , which ever value was higher.

\section{Questionnaire and data analysis}

Socio-demographic and risk factor data were gathered using a questionnaire that was pilot tested, and completed at the health facility during the patient's visit by trained interviewers. $\operatorname{IgM}$ and $\operatorname{IgG}$ serostatus was recorded as positive or negative, and seroprevalence was expressed as a percentage of the numbers sampled and tested. Exploratory analysis was performed to explore variables potentially associated with exposure to CCHF virus, including age, occupation, contact with animals (camels, donkeys, and goats), location, and gender. A multivariable logistic regression model was used to investigate risk factors associated with testing positive for anti-CCHFV antibody. Model reduction was based on likelihood ratio (LR) testing at a 5\% level. The analyses were performed using R v2.11.0 (R Development Core Team, 2010).

\section{Results}

\section{Patient demographics}

A total of 517 serum samples were collected from the patients presenting with febrile illnesses, including fevers of 
unknown origin, at Ijara Health Centre (248 samples) and Sangailu dispensary (269 samples), Ijara District, in North Eastern Kenya (Fig. 1). The sample consisted of $62 \%$ females and 38\% males aged between 5 and 90 years. The patients enrolled in the study presented with the following symptoms: fever $(98 \%)$, chills $(59 \%)$, cough $(34 \%)$, headache $(91 \%)$, joint aches $(79 \%)$, abdominal pain (52\%), and vomiting (30\%).

\section{IgM and IgG results}

The presence of both CCHF IgM and IgG antibodies were investigated among the sampled patients. An acute case (1/ 517) from Sangailu tested positive for anti-CCHF IgM, and 96 of 517 serum samples were positive for anti-CCHF IgG, of which $62(65 \%)$ were patients sampled from Sangailu, and 34 (35\%) from Ijara.

\section{Prevalence and risk factors for CCHFV}

Overall, 96 (19\%; 95\% CI 15,22\%) of the patients tested CCHF IgG antibody-positive. This proportion was significantly greater in Sangailu (23\%) than Ijara $(14 \% ; p=0.0063)$ and overall, males had a higher prevalence $(23 \%)$ compared to females (15\%). In Sangailu, 51.6\% of those testing positive were females and $48.4 \%$ males. In Ijara, $52.9 \%$ of those testing positive were females and $47.1 \%$ males. The results indicated that those testing positive clustered within the age range of 40-49 years (Fig. 2). In terms of occupation, farmers had the highest prevalence $(29.3 \%)$, followed by housewives $(18 \%)$, and businessmen (8\%). Contact with some animals increased the risk of CCHFV infection, with prevalence among those who had contact with goats, camels, and donkeys, observed at $18.8 \%, 20 \%$, and $20.2 \%$, respectively.

Multivariable logistic regression analysis precluded the need for correcting for contact with camels and goats (LR test $p=0.981$ and $p=0.4107$, respectively), and were therefore excluded from the final model. The final model results, presented in Table 1, indicated that age, location, and contact with donkeys were significantly associated with having antiCCHF IgG antibody. The results showed that the odds of $\mathrm{CCHF}$ increase by 1.02 for a 1-year increase in age after controlling for the other factors. The odds of being CCHF-positive

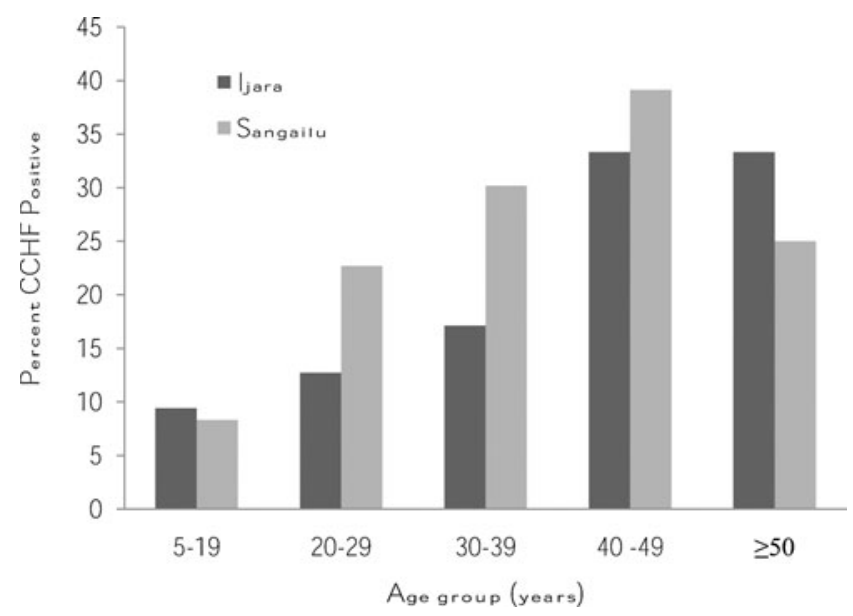

FIG. 2. CCHF IgG-positive individuals $(n=96)$ by age group in health facilities in Ijara and Sangailu, October 2010March 2011.
Table 1. Seroprevalence And Associated RisK FACTORS FOR CCHFV

\begin{tabular}{lccc}
\hline & $\begin{array}{c}\text { Anti-CCHF } \\
\text { IgG prevalence }\end{array}$ & OR $(95 \%$ CI) & $\begin{array}{c}\mathrm{p} \\
\text { Value }\end{array}$ \\
\hline $\begin{array}{l}\text { Age (years) } \\
\text { Sex }\end{array}$ & & $1.02(1.01,1.04)$ & $0.0038^{*}$ \\
$\quad$ Female & 15.0 & & \\
$\quad$ Male & 23.0 & $2.65(0.89,9.20)$ & 0.0960 \\
Occupation & & & \\
$\quad$ Farmer & 29.3 & & \\
$\quad$ Businessman & 8.0 & $0.30(0.02,1.72)$ & 0.2659 \\
$\quad$ Housewife & 18.0 & $1.58(0.50,5.77)$ & 0.4558 \\
$\quad$ Other & 9.4 & $0.60(0.24,1.40)$ & 0.2478 \\
Had contact & 20.2 & $1.92(1.05,3.72)$ & $0.0427^{*}$ \\
$\quad$ with donkeys & & & \\
Location & & & \\
$\quad$ Sangailu & 23.0 & & $0.0186^{*}$ \\
Ijara & 14.0 & $0.55(0.33,0.90)$ & 0 \\
\end{tabular}

*Significantly associated with being positive for anti-CCHF IgG antibody at the $5 \%$ level.

$\mathrm{OR}$, odds ratio; $\mathrm{CI}$, confidence interval.

CCHFV, Crimean-Congo hemorrhagic fever.

were higher in Sangailu than in Ijara (OR 0.55, 95\% CI $0.33,0.90)$. Individuals who had contact with donkeys were more likely to be CCHF-positive than those who did not (OR $1.92,95 \%$ CI 1.05,3.72). Although overall, a higher proportion of males than females tested positive for CCHF IgG, this difference was not statistically significant (OR 2.65, 95\% CI $0.89,9.20 ; p=0.096)$. Further analysis indicated that occupation of the subjects was also not significantly associated with being CCHF-positive (chi-square 4.1, $\mathrm{df}=3, p=0.25$ ).

\section{Discussion}

CCHF outbreaks constitute a threat to public health because of its epidemic potential, the severity of disease it causes, its potential to cause nosocomial outbreaks, and the limited options available for treatment and management of infected persons. Because CCHF is a zoonotic disease associated mainly with domestic animals, this study was designed to determine if the human population in Ijara District, North Eastern Kenya, which is essentially a pastoralist community living in a region where CCHFV has been detected among Hyalomma ticks, is getting exposed to CCHFV.

A single case out of the 517 patients with acute febrile disease tested positive for anti-CCHFV IgM. This was a female patient seen in Sangailu Health Centre, Ijara District, who had manifested with the following clinical signs: malaria smear-positive, fever $\left(37.8^{\circ} \mathrm{C}\right)$, cough, sore throat, headache, abdominal pain, dizziness, muscle aches, and joint pains. The outcome of this patient's disease is not known, as there was no follow-up of the sampled cases. This patient represents the first documented case of acute human CCHFV infection in the area. The patient had contact with goats and donkeys, which might have placed her at higher risk. A study carried out in Central Europe by Pak (1975) revealed that natural infection of large domestic mammals such as donkeys, cattle, camels, and horses, does occur. This indicates that donkeys elsewhere are potential reservoirs for CCHFV transmission, and this may be the case in the region we studied. 
The overall prevalence of CCHF IgG antibodies, which is indicative of prior exposure to CCHFV in the present study population, was $19 \%$. This is indicative of significant circulation of CCHF virus, not only among ticks, as was recently observed in the region (Sang et al. 2011), but also infecting the human population. The failure of the public health system to detect acute cases may be attributable to the non-specific nature of the clinical presentation, which makes it difficult for health care workers to differentiate CCHFV infection from other endemic causes of such febrile illness as malaria, brucellosis, and typhoid. The observed prevalence is fairly comparable to a survey conducted elsewhere among nomadic tribes in Senegal, where a seroprevalence rate of $13.1 \%$ was observed (Chapman et al. 1991).

The age group 40-49 years had the highest seroprevalence rate, which is comparable to the findings of a study carried out in Iran by Izadi (2004), and which confirms that important risk factors for CCHFV exposure include high-risk occupations (e.g., butchers, physicians, and veterinarians), having contact with livestock, and age over 40 years. In this study, the seroprevalence of CCHFV increased significantly with age, to a maximum of $36.23 \%$ at $40-49$ years old. This, however, contradicts a study carried out in nomadic populations in Senegal, where it was reported that the seroprevalence increased significantly with age to a maximum of 31.6\% among those 31-39 years old (Chapman et al. 1991), although the two age groups are adjacent. In another study in South Africa, CCHF IgG antibody prevalence among farmers increased with age (Fisher-Hoch et al. 1992). The high prevalence in the age group 40-49 years may be due to the fact that this is a mature age group, that most actively herds domestic animals, or actively handles them for purposes of trade or transportation (in case of donkeys), and are thus at a higher risk of contact with infected ticks and animal excrement. It is also possible that during seasonal migrations in search of pasture, they spend many days and nights outside providing protection for the other family members (the young and old) and their livestock against attack by bandits and wild animals, and thus risking contact with infected animals and hungry ticks.

The seroprevalence of CCHFV in Sangailu was significantly higher than that in Ijara. The population in Sangailu therefore appears to be at higher risk of exposure to CCHFV than the population in Ijara. Ijara (S01.57415 E040.12800) is closer to the Boni forest, and enjoys more humid conditions and rain than Sangailu (S01.27170 E040.66958), which is about $40 \mathrm{~km}$ away from Ijara, and has more arid conditions that are considered favorable for Hyalomma ticks, the primary vectors of CCHFV. The conditions prevailing in Sangailu may increase the risk of exposure due to increased virus activity among ticks or viremic animals.

Farmers were at higher risk than housewives and businessmen. Farmers in the pastoralist communities in North Eastern Province interact closely with their animals during milking, slaughtering, and caring for sick animals. During dry seasons, the farmers graze their livestock far from home, along the Tana River Delta and the Boni forest area, where water and pasture are abundant long after the rains have gone. In these habitats livestock from most parts of North Eastern Kenya and Somalia converge, and there are also potential interactions with wild animals, which increases the risk of transmission of CCHF virus from infected wild animals, which are believed to serve as reservoirs of CCHFV (EstradaPena 2001; Tonbak et al., 2006; Vorou et al. 2007).

Housewives were the second most affected risk group. This could be through participation in high-risk activities such as taking care of livestock, especially small animals (sheep and goats), which involves milking and assisting in slaughtering animals. This finding is consistent with studies carried out in Africa, Europe, and the Middle East, by Heymann (2004), and Izadi and associates (2004), which showed that people working with animals, such as farmers living in endemic areas, medical personnel, veterinarians, abattoir workers, shepherds, animal workers, butchers, and tanners, have the highest prevalence of $\mathrm{CCHF}$, indicating their high risk of infection (Heymann 2004; Izad et al. 2004).

Other factors, including age, location, and contact with donkeys, were also shown to be risk factors for exposure to CCHFV. The fact that donkeys are widely used in the study area for domestic purposes as a means of transportation of wares including grains and other foodstuffs, commercial commodities to the market for sale, and the movement of water for domestic use over long distances, increases human/ donkey contact, and thus the risk of CCHFV transmission. These animals are rarely slaughtered, and because of their limited commercial value coupled with the fact that they do not die from tick-borne diseases, donkeys have low priority during acaricide treatment of livestock. This could make them a good source of tick infestations, and thus the potential of carrying infectious ticks. Enhancing community awareness of their potential role in the spread of tick-borne diseases is a simple means of reducing the transmission of CCHFV. A study by Shimshony (2010) found CCHF antibodies in sera from horses, donkeys, goats, cattle, sheep, and pigs in various parts of Europe, Asia, and Africa.

\section{Conclusions}

This study confirms the transmission of CCHF virus to the human population in Ijara District. Age, location, and contact with donkeys, are all risk factors for CCHF virus exposure, with Sangailu being more at risk than Ijara. Health care providers should be informed of the prevalence of CCHF in the area, and be equipped with clear case definitions to identify possible cases, and have the therapies and resources needed to care for CCHFV patients, including infection control measures and medical equipment that are often lacking in rural hospitals, in an effort to empower them to deal with CCHFV infections. The community should also be educated about the risks of the disease, and how to avoid infection through tick control, the avoidance of high-risk activities, and effective animal husbandry, and to control tick infestations on all domestic animals including donkeys. Further research is recommended to investigate the possible role of goats, donkeys, and other potential animal reservoirs of disease in the tickCCHF life cycle. This will assist in focusing control efforts and understanding CCHFV epidemiology in North Eastern Kenya. There is also a need to better characterize the clinical presentation of this infection in this population, in order to improve detection and control of the disease.

\section{Acknowledgments}

We thank the families of Ijara District who participated in the study, and the tremendous work of Sangailu dispensary 
and Ijara Health Centre staff (Mr. Saidi, the District Chief Laboratory Technologist, Ijara; Dr. Sonde, the District Medical Officer of Health and the District Health Management Team, Ijara District) for their support. We also thank the Arbovirus Incidence and Diversity Project consortium-icipe, ILRI, KARI, the Kenya Ministry of Livestock-Department of Veterinary Services, KEMRI, the Kenya Ministry of Public Health and Sanitation and KWS; and the director of the International Centre for Insect Physiology and Ecology, Prof. Christian Borgeimester, for providing the necessary environment and support for this work. This research was funded by Google .org, the philanthropic arm of Google.

\section{Author Disclosure Statement}

No competing financial interests exist.

\section{References}

Casals J. Antigenic similarity between the virus causing Crimean hemorrhagic fever and Congo virus. Proc Soc Exper Biol Med 1969; 131:233-236.

Chapman LE, Wilson ML, Hall DB. Risk factors for CrimeanCongo hemorrhagic fever in rural northern Senegal. J Infect Dis 1991; 164:686-692.

Dunster L, Dunster M, Ofula V, et al. First documentation of human Crimean-Congo hemorrhagic fever, Kenya. Emerg Infect Dis 2002 Sept. http://www.cdc.gov/ncidod/EID/ vol8no9/01-0510.htm

Elata TA, Elageb MR, Karsany SM, et al. A nosocomial transmission of Crimean-Congo hemorrhagic fever to an attending physician in north Kordufan, Sudan. Virol J 2011; 8:303.

Ergonul O. Crimean-Congo haemorrhagic fever. Lancet Infect Dis 2006; 6:203-214.

Estrada Pena A. Forecasting habitat suitability for ticks and prevention of tick-borne diseases. Vet Parasitol 2001; 98:111-132.

Fisher-Hoch Sp, McCormick JB, Swenpopel R, et al. Risk of human infections with Crimean-Congo hemorrhagic fever virus in South Africa rural community. Am J Trop Med Hyg 1992; 47:337-345.

Gao X, Nasci R, Liang G. The neglected arboviral infections in mainland China. PLoS Negl Trop Dis 2010; 4:e624. doi:10.1371/journal.pntd.0000624

Harun A, Emre O, Mitat K. An antigenic investigation of Crimean-Congo hemorrhagic fever virus (CCHFV) in hard ticks from provinces in northern Turkey Trop. Anim Health Prod 2010; 42:1323-1325. DOI 10.1007/s11250-010-9579-1
Heymann DL. An official report of the American Public Health Association. In: Control of Communicable Diseases Manual. Heymann DL, ed. Washington, DC: American Public Health Association, 2004:35-37.

Hoogstraal H. The epidemiology of tick-borne Crimean Congo haemorrhagic fever in Asia, Europe and Africa. J Med Entomol 1979; 15:307-317.

Izadi S, Holakouie-Naieni K, Madjdzadeh SR, et al. CrimeanCongo hemorrhagic fever in Sistan and Balouchestan Province of Iran, a case-control study on epidemiological characteristics. Int J Infect Dis 2004; 8:299-306.

Nichol ST. Bunyaviruses. In: Fields Virology. Knipe DM, Howley PM, eds. Philadelphia: Lippincott Williams \& Wilkins, 2001:1603-1633.

Pak TP. Structure of the distribution of Crimean-Congo hemorrhagic fever. Tadzhikistan. Mater 9 Simp Ekol Virus (Dushanbe, October 1975) 1095; 39-43.

R Development Core Team. R: A language and environment for statistical computing. R Foundation for Statistical Computing, Vienna, Austria, 2010; ISBN 3-900051-07-0, http://www .R-project.org

Sang R, Lutomiah J, Koka H, et al. Crimean-Congo hemorrhagic fever virus in hyalommid ticks, northeastern Kenya. Emerg Infect Dis 2011. http://www.cdc.gov/EID/content/17/8/ 102064.htm

Shimshony A. Crimean-Congo hemorrhagic fever virus infection A re-emerging, potentially deadly illness. Infectious Diseases News Educational Lab: Zoonotic Infections, 2010; 08:50:7. http://www.infectiousdiseasenews.com/article/64303.aspx

Swanepoel R. Exotic Viral Infections in Nairovirus Infections. Porterfield JS, ed. London, 1995.

Tonbak S, Aktas M, Altay K, et al. Crimean-Congo hemorrhagic virus: genetic analysis and tick survey in Turkey. J Clin Microbiol 2006; 44:4120-4124.

Vorou R, Pierrout IN, Maltazou HC. Crimean-Congo hemorrhagic fever. Curr Opin Infect Dis 2007; 20:495-500.

Address correspondence to: Olivia Wesula Lwande Department of Human Health International Centre for Insect Physiology and Ecology P.O. Box 30772

Nairobi 00100

Kenya

E-mail: olwande@icipe.org 